Demystifying Spiritual Care: An Interprofessional Approach for Teaching Residents and Hospital Chaplains to Work Together

\section{Setting and Problem}

Residents often face concerns that relate to patients' spirituality, especially when caring for them in the inpatient setting. The range of issues confronted is wide, from consent for medical procedures to end-oflife decision making and addressing the emotional distress that commonly arises when dealing with acute and chronic medical illness. Many patients draw on spiritual beliefs, practices, and communities to find strength, create meaning from illness, and make medical decisions. Residents rarely receive training in spiritual assessment and may miss opportunities to understand and address patients' pressing concerns. Similarly, chaplains (professionals hired by hospitals to provide spiritual support to patients) are not routinely trained via direct clinical experience to work closely with physicians.

To improve education for both professions, we created a team-based curriculum for chaplain trainees and internal medicine residents working side-by-side in the inpatient setting. An initial needs assessment survey of residents showed that although $82 \%$ (28 of 34) reported that addressing a patient's spirituality was an important part of patient care, they also reported barriers, including lack of time, uncertainty around knowing whether and when to address spirituality, and difficulty finding appropriate language. We found that only $21 \%$ (7 of 34 ) of residents had knowledge of a spiritual assessment tool, and only $9 \%$ (3 of 34 ) had used one.

\section{Intervention}

To address perceived gaps in the knowledge and skills for addressing patients' spiritual needs, we developed

DOI: http://dx.doi.org/10.4300/JGME-D-15-00637.1

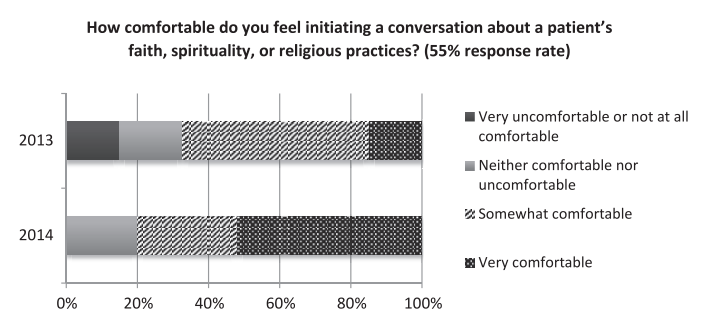

Note: ANOVA $P$ value $=.01$

On your last month on an inpatient service, how many times did you make a chaplain referral?

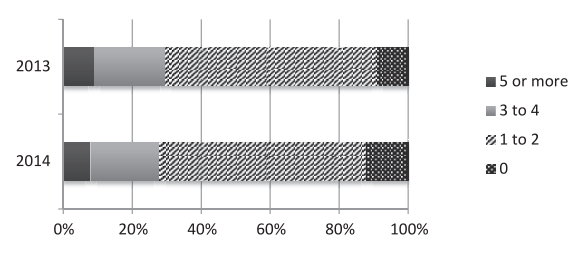

Note: ANOVA $P$ value $=.92$

FIGURE

Resident Survey From Before and After Curriculum Intervention

an interprofessional curriculum at Johns Hopkins Bayview Medical Center, integrating a chaplain intern with 1 inpatient medical team. Chaplain interns in clinical pastoral education are masters-level theology students receiving supervised clinical training. After completing an 8-month curriculum development process by a team of physicians and chaplains and conducting a pilot, we implemented the full curriculum beginning in fall 2013.

The curriculum encompasses 3 distinct phases: orienting, rounding, and partnering. In orienting, a chaplain educator introduces medical residents to the FICA $^{1}$ (a spiritual assessment tool) and to the roles of hospital chaplains. Chaplain interns are oriented by an internal medicine attending physician to inpatient rounding and team member roles. For the rounding phase, chaplain interns participate in the medical team's rounds and share short didactics on chaplains' roles and spiritual assessment. During the partnering phase, chaplain interns and residents collaborate on patient care issues that were identified during rounds. During the 4-week rotation, chaplain interns work with the team of medical residents 1 day per week. The curriculum also provides opportunities for structured reflection around meaningful patient encounters. We encourage medicine attending physicians to regularly include chaplain interns in discussions during rounds.

\section{Outcomes to Date}

During implementation in 2013-2014, we examined residents' ratings from each inpatient rotation on the medicine wards. We compared ratings from 30 residents who had completed evaluation of both 
standard $(\mathrm{n}=120)$ and intervention $(\mathrm{n}=34)$ rotations. Interprofessional rotations with chaplains received significantly higher ratings than standard rotations in 2 domains of spiritual care: (1) residents' understanding of patients' values, and (2) level of collaboration with chaplains. These findings suggest a positive impact on residents' patient care experience.

In spring 2014, following implementation of the curriculum, medical residents were resurveyed, and their responses were compared to the 2013 needs assessment. There was a $36 \%$ absolute increase in the percentage of residents who reported being very comfortable in discussing a spiritual concern with a patient (FIGURE). No residents reported that having chaplains participate in care took too much time, and $21 \%$ (7 of 34 ) wished that there was more time. In both surveys, more than $85 \%$ (29 of 34 ) of residents reported having made at least 1 chaplain referral during their most recent month of inpatient service. Other faculty members (from psychiatry, pediatrics, and anesthesiology) at Johns Hopkins University have proposed expanding the curriculum to their residents.

This is a novel, feasible, effective approach for teaching residents to address patients' spiritual needs by partnering with chaplaincy training programs that exist at many academic institutions.

\section{Patrick Hemming, MD, MPH}

Medical Instructor, Division of General Internal Medicine, Department of Medicine, Duke University School of Medicine

\section{Paula Teague, DMin, MBA}

Senior Director, Spiritual Care and Chaplaincy, Johns Hopkins Health System

\section{Ty Crowe, MDiv}

Director of Spiritual Care and Chaplaincy

Department, Johns Hopkins Hospital

\section{R. B. Levine, MD, MPH}

Associate Professor, Division of General Internal Medicine, Department of Medicine, Johns Hopkins University School of Medicine

Corresponding author: Patrick Hemming, MD, MPH, Duke University School of Medicine, 4220 North Roxboro Street, Durham, NC 27704, 919.680.1290, patrick.hemming@duke.edu

\section{References}

1. The George Washington University Institute for Spirituality \& Health. FICA Spiritual Assessment Tool. http://smhs.gwu.edu/gwish/clinical/fica. Accessed March $16,2016$. 